\title{
Weighing Strategy Based on Dynamic Signal Processing Technology
}

\author{
Mutian Zhu ${ }^{1, \text { a }}$ \\ ${ }^{1}$ College of Electric and Electronic Engineering, Zibo Vocational Institute, Zibo 255314, China \\ a Email: zbvczmt@163.com
}

Keywords: signal correlation, normalized LMS, cancel noises, fuzzy partition.

\begin{abstract}
Loaders weighing process is affected by the lifting position, acceleration, and mechanical vibration and other factors, the measurement accuracy of the conventional way is difficult to improve. This paper proposes a set of estimation algorithm which uses the correlation between the weighting measuring signal and the carrier-free signal in the same state. This paper faintly differentiates many kinds of noises to lift acceleration and adopts a methods based on normalized LMS to estimate the adaptation of two kinds of signals so as to cancel noises. In this way, the measuring accuracy is improved.
\end{abstract}

\section{Introduction}

Through the establishment of force transmission to the mathematical model, the use of the fuel tank pressure to calculate the bucket load of cargo weight in the dynamic weighing of loader, is a commonly used dynamic weighing. In this way is simple and reliable, but measurement accuracy is very rough, according to the test caused the error mainly from the following aspects:(1) Tests show that when a loader is hoisting a certain weight of materials, the value of pressure in actuating arm $(\mathrm{P})$ is varying and the relationship between $\mathrm{P}$ and the stroke of piston rod (L) is not fully linear. (2)Affected by acceleration of gravity, different lifting speeds impact on gravity in the range of $-14 \% \sim 10.4 \%$, and thus the noises are created in dynamic weighing. (3)When the engine works, in unknown system mechanical variation causes disturbance which has the same frequency as the useful signal (about $3-25 \mathrm{~Hz}$ ) and is an important fact to affect the accuracy. These signals are mixed together to lower the SNR (signal to noise ratio) and adversely impact on the weighing accuracy.

The equipment structure of the loader is normative in the same condition, so the function between load capacity and the pressure in hoisting hydraulic cylinder of the actuating arm is definite. The noises and signals are considered as random signals. Automatically adjust and update the filter parameters in the present moment by using previous time filter parameter values, adapt to the unknown signal and noise statistical properties of the optimal filtering can achieve satisfactory results.

\section{The basic structure of the adaptive filter}

Digital filter implementation of digital filters can be composed of two parts: First, filter processing, to borrow a set of taps coefficients to calculate the output of the filter with the comparison output and expected output, resulting in an estimated error. Second, adaptive processing algorithm, based on the estimated error automatically adjusts the filter tap coefficients of the right to re-. The structure of the adaptive filter is FIR transverse structures, as in Fig. 1. 


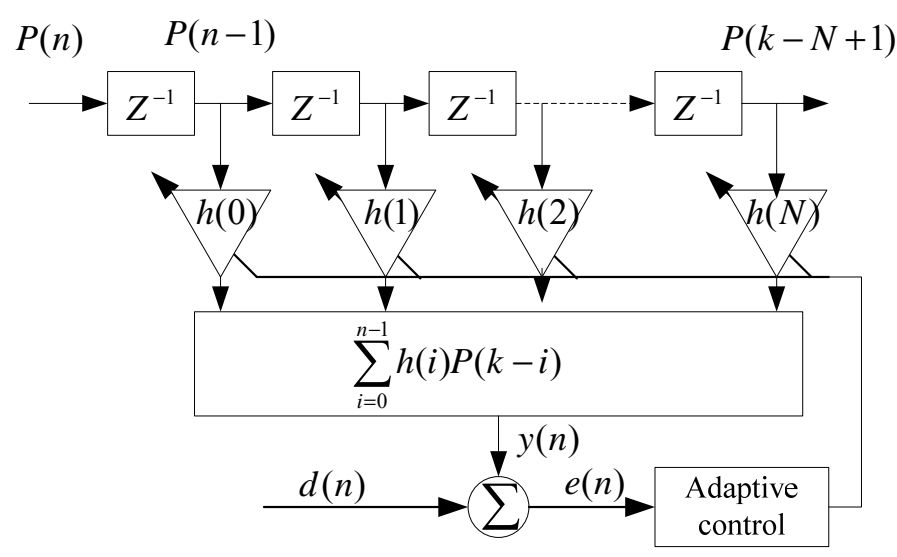

Figure 1 Adaptive transversal filter structure

There into, $d(n)$ is the expected value signal, $y(n)$ is filter output, use the error signal by using is $e(n)=d(n)-y(n)$, estimated value, constitutes the error signal by the input signal vector $P(n)$ and the filter coefficient vector $h(n)$ at the moment $n$ :

$$
e(n)=d(n)-p^{T}(n) * h(n)
$$

The filter design principle is to make $e(n)$ tends to the minimum mean square error minimum (of MMSE)-based optimization principles have:

$$
\begin{aligned}
& \varepsilon=E\left[e^{2}(n)\right]=E\left[d^{2}(n)\right]-2 E\left[d(n) p^{T}(n)\right] h(n)+h^{T}(n) E\left[p(n) p^{T}(n)\right] h(n) \\
& =\sigma_{d}{ }^{2}+2 \tau^{T} h+h^{T} R h
\end{aligned}
$$

In this formula, $\sigma_{d}{ }^{2}=E\left[d^{2}(n)\right]$ is the variance, $\tau=E[p(n) d(n)]$ is cross-correlation vector of $P(n)$ and $d(n) . R=E\left[p(n) p^{T}(n)\right]$ is the autocorrelation matrix of $P(n) . \varepsilon$ is a quadratic function of the filter coefficients, the partial derivative of $h(n)$ there exists a unique external $\varepsilon$ tends to the smallest.

$\mathcal{E}$ is a quadratic function of $h$, which will form the performance-surface as a bowl. Down to adjust the weight vector, the minimum point of the search performance surface, performance-surface, the steepest descent direction is the surface of the negative gradient direction along the direction of performance-surface steepest. The steepest descent method is an iterative search process using a recursive method to calculate the filter coefficient vector update estimates, $\mu$ for the control of the convergence rate constants, called the compensation parameters.

$$
h(n+1)=h(n)-\mu^{*} p(n) * e(n)
$$

Time index $n$ increased by 1 , return to the formula (1) (2) Repeat the above steps until the steady state so far.

The condition of convergence of LMS is:

$0<\mu<2 / \lambda_{\max }$

$\lambda_{\max }$ is the maximum of correlation matrix.

The renewed iterative formula of normalization LMS algorithm, as in [1]:

$$
h(n+1)=h(n)+\frac{\mu}{\gamma+p^{T}(n) p(n)} e(n) p(n)
$$

Where $\mu$ is the fixed convergence factor for control disorder, parameter $\gamma$ is set for $p^{T}(n) p(n)$, and convergence factor satisfies the following function:

$$
0<\mu(n)=\frac{\mu}{2 \operatorname{tr}[R]}<\frac{1}{\operatorname{tr}[R]} \text { or } 0<\mu<2
$$

Formula (4) and (5) form normalization LMS algorithm, whereas $0<\gamma<1$. 


\section{System design}

The pressure signal, which is also be effected by acceleration in the hoisting operation of the load,will fluctuate owing to the differences between different kinds of loaders and models of the same kind of loader. Therefore, the error of measuring weight load in the same model is quite great. Figure 1 shows the relationship between the full range of hydro-cylinder in the actuating arm and sampling value of oil pressure. Because of the existing angular acceleratione, the curve $\mathrm{P}-\mathrm{L}$ shows non-linear. In most of stroke, the curve shows in linear progression and these three curves are parallel. From the beginning to end of $\mathrm{L}$, the nonlinearities facts are seriously, so in order to get effective sampling values, the area sampling of measuring device is set in the process of loader ascension. In this area, the relative stability of pressure signals is required, so the collected data has low degree of difference.

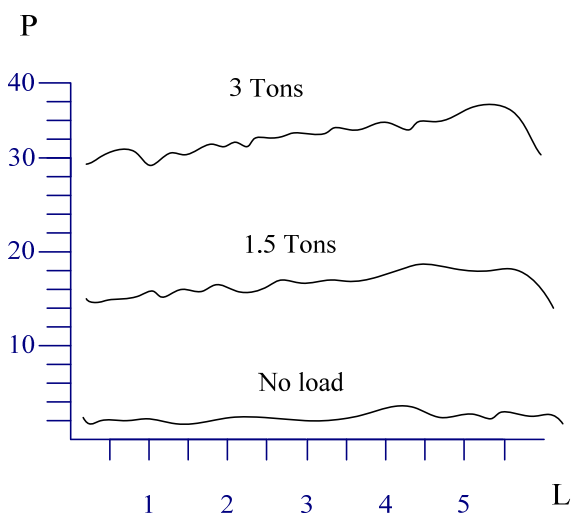

Figure 2 P-L curve

Measure the actual oil pressure $P(n)$ which is also as noisy signal and original signal. Put $P(n)$ and reference signal $N_{1}(n)$ together do countervailing arithmetic to cancel the noise in useful signals. The key point lies in the reference signal has correlation with signals waiting to eliminate noise, and no-correlation with signals waiting to draw. Its functional block diagram is shown as follows:

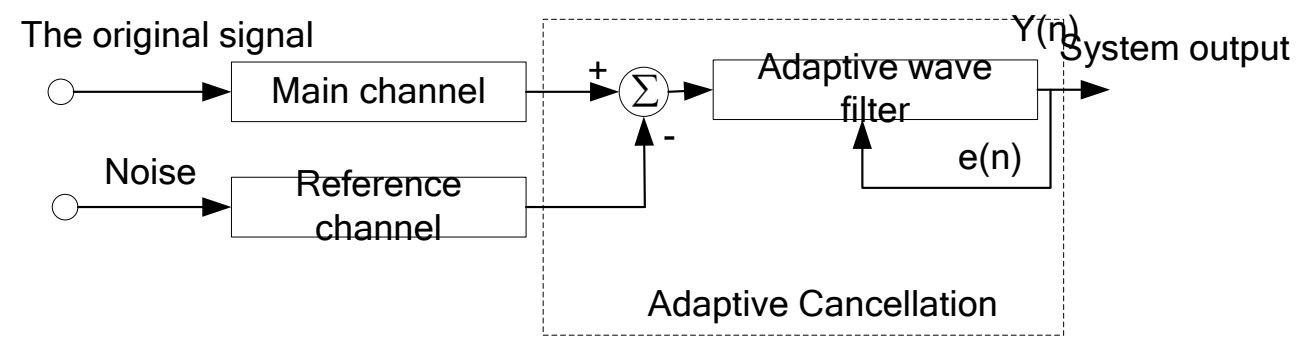

Figure 3 Noise countervailing

The original and noise signals become the original inputs by main channel: $P(n)=P_{0}(n)+N_{0}(n)$ ). Noise becomes another input signal $N_{1}(n)$ when passing the reference channel. $P_{0}(n), N_{0}(n)$ and $N_{1}(n)$ are stationary zero-mean random sequence. Since $N_{0}(n)$ and $N_{1}(n)$ are from the same noise source, there is some certain correlation between them. But $N_{0}(n), N_{1}(n)$ and $P_{0}(n)$ are not related, their difference can cancel noises effectively.

In the real dynamic weighting process, the simplest way to get reference noise $N_{1}(n)$ is to imitate another weighting system which is synchronous with the actual one, and this means the loader is idling running. The machinery and hardware of analog weighting system has no difference with the actual one. Its transmitter output can be considered as the reference noise input. But the cost is too high to realize it in real life. So a section of address space to store different angular accelerations is opened in the lower controller. The loader with no-load is hoisting to collect data in sampling area as 
the reference input. System vibration information in this data, together with original noise, has some correlation. In view to the different lift speed having great impact on weighting, we collect and store original noise $N_{0}(n)$ on speed.

Faintly differentiate five sectors $\left\{N_{0}(0), N_{0}(1), \ldots \ldots, N_{0}(5)\right\}$. As the same sampling period, the sampling number can measure the hoisting speed when the loader passes through the sampling area. The data of this section can be updated real-timely. The loader is found no-load when it passes through weighting section, the data in this section can be regarded as the newest reference noise.

\section{System implementation and simulation}

Program initialization, including the identification of the filter order $\mathrm{M}$, the convergence factor, the initial tap vector $h(0)$.

1) Calculate the moment $\mathrm{k}$ of the filter output $\mathrm{y}(\mathrm{k})$ and error output $\mathrm{e}(\mathrm{k})$.

2) According to the iterative formula to calculate the next time $\mathrm{h}(\mathrm{k}+1)$.

3) Let $\mathrm{k}=\mathrm{k}+1$, go to step 1 .

$\mu=0.18, \gamma=20$ in formula (4) reaches comparably satisfying results after over 20 times tests. In different loads, the oil pressure in hydraulic-cylinder of actuating arm of the loader keeps a petty proportionate relationship, so we can demarcate curve first. By testing no-load operation, we get a P-L curve and collect the data on this curve. And then we get the actual oil-pressure curve of hydraulic-cylinder of actuating arm from the material staying to weight (two standard mass, such as 2 and 3 tons). Next three sets of data do noise countervailing by the normalization LMS algorithm. In this way, we can calculate the weight of material, get high measuring accuracy. This method does not have much calculation and has a very good feasibility. The specific data is as follows:

Table 1 Data test

\begin{tabular}{cccc}
\hline Standard, $[\mathrm{Kg}]$ & Actual measurement, $[\mathrm{Kg}]$ & Times of sampling & Deviation, [\%] \\
\hline 2000 & 1991 & 21 & 9 \\
2000 & 1996 & 28 & 4 \\
2000 & 2003 & 33 & -3 \\
2000 & 2012 & 45 & -12 \\
2000 & 2006 & 43 & -6 \\
2000 & 1998 & 32 & 2 \\
2000 & 1994 & 30 & 6 \\
2000 & 2001 & 34 & -1 \\
3000 & 3002 & 38 & -2 \\
3000 & 3008 & 36 & -8 \\
3000 & 3014 & 45 & -14 \\
3000 & 2989 & 22 & 11 \\
3000 & 2998 & 27 & 2 \\
3000 & 2996 & 26 & 4 \\
3000 & 2994 & 22 & 6 \\
3000 & 2999 & 40 & 1 \\
\hline
\end{tabular}

In this chart, times of sampling means the actual times in sampling area, because the sampling period is constant, this index indicate the hoisting speed of actuating arm of the loader.

\section{Conclusions}

The disturbances on loader are complicated, and filtering is an effective means to barrage jamming. By designing useful signals to get through the noise countervailing system, we can draw useful signals and restrain noise signals. By the standard weight, the applied 30-type loader reaches a high measurement standard. In real engineering application since the poses of loading shovel in loaders are 
different and the working places of loaders are not in absolute surfaces, this will cause great errors. How to overcome the error is the further research direction of our issue.

\section{References}

[1] Shigeji I, Akihiko S. An Adaptive Noise CancellerWith LowSiginal Distortion for Speech Codes [J]. IEEE Trans on Signal Processing, 1999, 47(3): 665-673.

[2] Chang Yilin,Wang Fusheng \&Li Bingbing etc. A Variable Step Adaptive Algorithm in Echo Cancellers [J], Journal of Xi' an University, 1990, 17(2): 30-34.

[3] Simon H. Adaptive Filter Theory [M]. Beijing: Publishing House of Electronics Industry, 1998. 433.

[4] H.B. Bahar. Dynamic weight estimation using an artificial neural network. Artificial Intelligence in Engineering, 1998,12(4):135-139.

[5] Yu Zhang,Ming xin Yuan and Hao Chu. The Research Thought and Signal Analysis on the Automobile Weigh-in-Motion (WIM) Device. ICTTS,2002. 